\title{
MULTISCALE LIQUID METAL THIN-FILM PATTERNING BASED ON SOFT LITHOGRAPHY FOR SKIN-MOUNTABLE, SOFT AND 3D-INTEGRATED BIOLOGICAL MICROSYSTEMS
}

\author{
Min-gu Kim, Choongsoon Kim, Hommood Alrowais, Patrick Getz, and Oliver Brand \\ School of Electrical and Computer Engineering, Georgia Institute of Technology, GA, USA \\ Institute for Electronics and Nanotechnology, Georgia Institute of Technology, GA, USA
}

\begin{abstract}
This paper presents multiscale liquid metal (eutectic gallium-indium alloy, EGaIn) thin-film patterning by utilizing an additive stamping approach for large-scale $(\mathrm{mm}-\mathrm{cm})$ soft electronics and a subtractive reverse stamping approach for micro-scale $(\mu \mathrm{m}-\mathrm{mm})$ soft electronics. By combining these complementary patterning techniques, fabricated soft electronic components can be vertically integrated to form high-density and multifunctional soft microsystems. To highlight the fabrication and integration capabilities, a skin-mountable, soft and 3D-integrated biological microsystem, comprising a soft sensing layer and a soft circuit layer, is demonstrated for non-invasive and real-time heart rate and blood oxygen monitoring.
\end{abstract}

\section{INTRODUCTION}

Progress in soft materials and associated manufacturing technologies has enabled wearable and skin-mountable electronics for various sensing applications [1-3]. In this regard, the use of intrinsically soft conductors, such as gallium-based liquid metal (eutectic gallium-indium alloy, EGaIn), is promising because of its non-toxicity and favorable mechanical and electrical $\left(\sigma=3.4 \times 10^{6}\right.$ $\mathrm{S} / \mathrm{m})$ properties $[4,5]$. The low melting temperature $\left(\mathrm{MP}<15^{\circ} \mathrm{C}\right)$ and negligible vapor pressure of EGaIn enable room-temperature and ambient pressure material processing [6]. Moreover, thanks to the formation of a thin oxide layer $(t \approx 1-3 \mathrm{~nm})$ on the EGaIn surface under atmospheric oxygen level, EGaIn structures maintain their mechanical shape, allowing 2D/3D EGaIn patterns on soft elastomeric substrates, such as poly(dimethylsiloxane) (PDMS) [7].

The moldable characteristics of EGaIn have enabled a range of patterning methods based on lithography-assisted printing [8, 9], fluid injection $[10,11]$, as well as additive $[12,13]$ and subtractive $[14,15]$ direct-write processes. Lithography-assisted stencil printing yields simple and high-throughput EGaIn patterning on elastomeric substrates [8]. However, limitations of this approach are the relatively low resolution, resulting rough EGaIn surfaces, and excessive EGaIn loss during the stencil lift-off process. Subtractive direct patterning techniques enable an inexpensive and facile approach to pattern fine EGaIn lines, but the serial process makes EGaIn removal slow in the case of patterning small EGaIn features on large substrates [15]. 2D/3D direct writing techniques allow to deposit EGaIn patterns in desired locations only, but the resolution is limited because of the size limitation of the injection nozzles [12, 13]. Microfluidic injection and vacuum filling approaches provide better resolution, but the used microchannels require relatively large thicknesses to reduce pressure drops [10]. Using a microtransfer deposition process, the smallest EGaIn features so far with width (w) $>2 \mu \mathrm{m}$ were demonstrated, but this technology suffered from EGaIn residues formed outside of the channel areas during the molding process [16]. Therefore, creating smooth and uniform EGaIn thin films in combination with high resolution and size scalability remains challenging for high-density and multifunctional soft microsystems $[17,18]$.
Soft lithography $[19,20]$ with wetting/non-wetting surface modifications in combination with 3D integration can potentially solve current EGaIn patterning challenges, by providing i) smooth and uniform EGaIn thin-film deposition, ii) multiscale patterning, and iii) high-density and functional integration. Recently, we demonstrated high resolution, uniform, and residue-free EGaIn thin-line patterning ranging from single micrometer to millimeter scales by utilizing a chemical surface modification and residue transfer process in a reverse stamping approach for soft microelectronic devices [21, 22]. Building on our previous work [21-25], this paper presents multiscale EGaIn thin-film patterning by utilizing an additive stamping process for large-scale $(\mathrm{mm}-\mathrm{cm})$ soft electronics and the subtractive reverse stamping process for micro-scale $(\mu \mathrm{m}-\mathrm{mm})$ soft electronics, as shown in Figure 1 (a). By combining these complementary patterning techniques, soft electronic components built with different patterning processes can be integrated to form high-density and multifunctional soft microsystems.

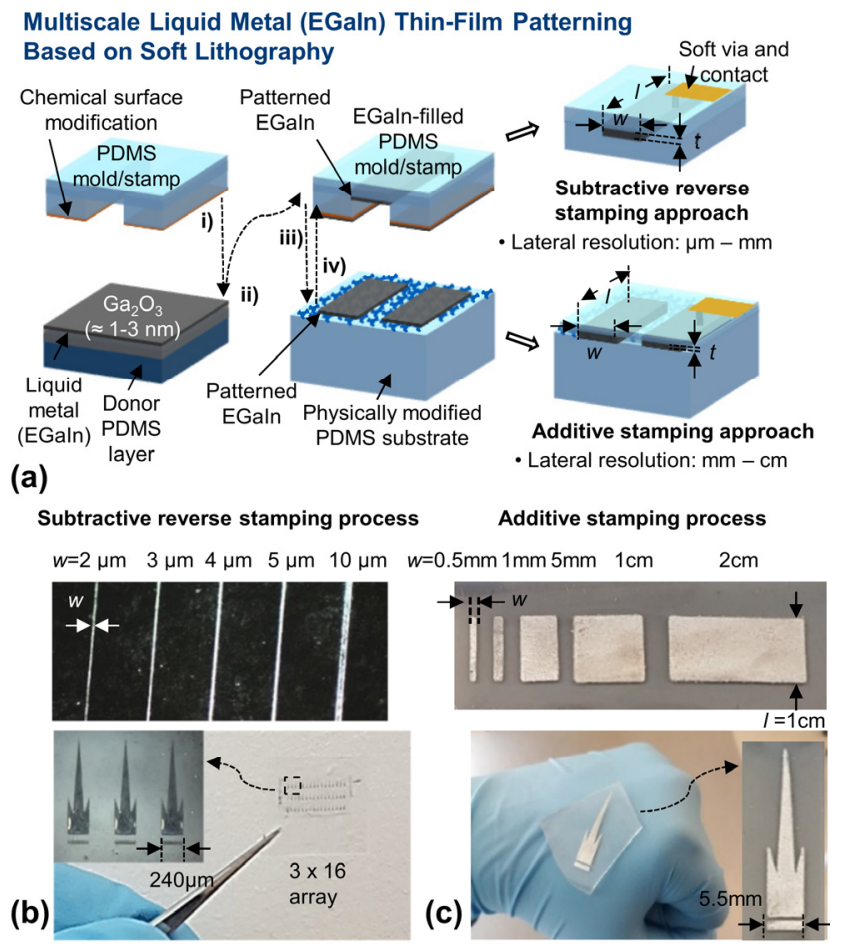

Figure 1: (a) Illustration of complementary multiscale liquid metal (eutectic gallium-indium alloy, EGaIn) thin-film patterning techniques with (b) micro-scale and high-density EGaIn lines and Georgia Tech logos embedded in chemical-surface-modified PDMS molds fabricated using a subtractive reverse stamping process and (c) centimeter-scale and large-area EGaIn patterns and Georgia Tech logo on physical-surface-modified PDMS substrates fabricated using an additive stamping process. 


\section{DESIGN AND FABRICATION}

Multiscale Liquid Metal Thin-Film Patterning: Subtractive Reverse Stamping and Additive Stamping Processes

Figure 1 (a) illustrates the two complementary multiscale EGaIn thin-film patterning techniques based on soft lithography: (1) a subtractive reverse stamping process relying on chemical surface modification for micro-scale and high-density EGaIn patterns embedded in PDMS microchannels and (2) an additive stamping process for centimeter-scale and large-area EGaIn patterns relying on a physical-surface-modified PDMS substrate. Thereby, physical and chemical surface modifications provide proper wetting and non-wetting surfaces, which enable fine, uniform, and residue-free EGaIn thin films. The chemical surface modification using toluene increases the hydrophobicity of PDMS surfaces, which minimizes EGaIn residue formation and allows them to be readily transferred to a sacrificial PDMS layer. Using the reverse stamping process, molded EGaIn in pre-defined PDMS microchannels showed high-resolution, residue-free, and uniform lines (Figure 1 (b)). On the other hand, the physical surface modification creates a paper-textured PDMS surface, increasing the surface area and ultimately enhancing wettability to form uniform and smooth EGaIn thin films using the additive stamping process (Figure 1 (c)).

In both cases, the fabrication process starts with the selective chemical surface modification of either a PDMS mold or PDMS stamp (optional process) (Figure 1 (a)-i)). The PDMS mold/stamp is then pressed onto a donor PDMS substrate coated with EGaIn and separated from it (Figure 1 (a)-ii)). During the molding process, EGaIn adheres to all surfaces of the PDMS mold/stamp, including concave channels and protruding surface areas. In the next step, the PDMS mold/stamp wet with EGaIn can be reverse stamped to remove EGaIn residues on the protruding surfaces or additive stamped to deposit EGaIn films on the physical-surface-modified PDMS substrate (Figure 1 (a)-iii) and -iv)).

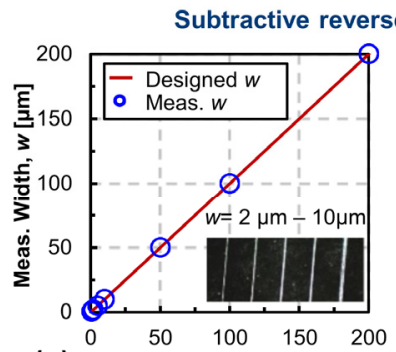

(a) Designed Width, $w[\mu \mathrm{m}]$

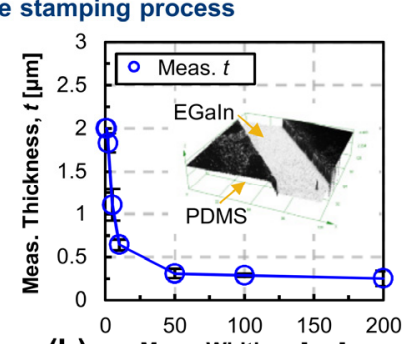

(b)
Meas. Width, $w[\mu \mathrm{m}]$
Figure 2: Subtractive reverse stamping process: (a) measured EGaIn width as a function of the designed PDMS mold width and (b) resulting EGaIn thickness as a function of the patterned EGaIn width.
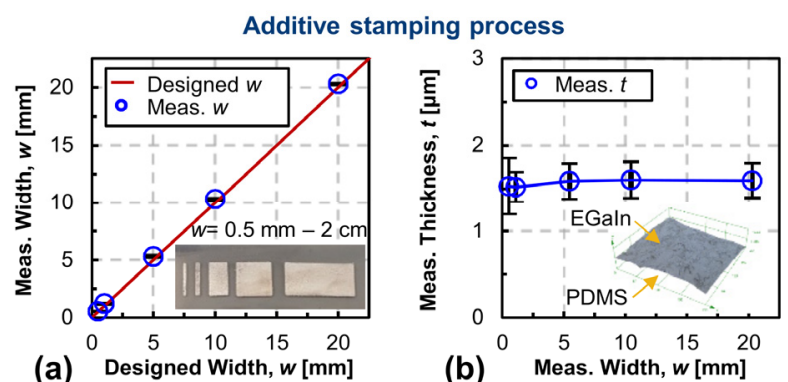

Figure 3: Additive stamping process: (a) measured EGaIn width as a function of the designed stamp width and (b) resulting film thickness as a function of the patterned EGaIn width.
As demonstrated in our previous research [21, 22], the subtractive reverse stamping process yields fine, uniform, and thin EGaIn lines inside of the PDMS channels without residues on the surrounding protruding surfaces, achieving a lateral line width resolution as small as $w=2 \mu \mathrm{m}$ at film thicknesses of $t \approx 1.8 \mu \mathrm{m}$, as shown in Figure 2. The subtractive approach showed size scalability from $2 \mu \mathrm{m}$ to $1-2 \mathrm{~mm}$ in line width. However, for channel widths $>2$ $\mathrm{mm}, \mathrm{EGaIn}$ is not effectively transferred to the inside of the PDMS channels because of the sagging deformation of wide channels during the pressure-based molding process. Therefore, the pattern dimensions using the subtractive reverse stamping approach are limited to the single $\mu \mathrm{m}$ to a couple of $\mathrm{mm}$ scale.

In contrast, the additive stamping approach provides large-area EGaIn thin-film patterning (Figure 3). In this process, a PDMS stamp wet with EGaIn is stamped onto a paper-textured PDMS substrate. Thereby, the paper texture, which comprises randomly distributed micro cellulose fiber structures, is transferred to a PDMS surface using a conventional PDMS casting method [26]. The resulting paper-textured PDMS exhibits a surface microstructure, which allows it to better wet with EGaIn compared to a normal, smooth PDMS surface (Figure 4). As a result, continuous EGaIn films can be transferred to paper-textured PDMS with multiple stamping steps. The smallest stamped EGaIn feature size was $w=$ $500 \mu \mathrm{m} / t \approx 1.5 \mu \mathrm{m}$, as shown in Figure 3. While this additive stamping process shows relatively poor lateral resolution, it is very suitable for large-area patterning and thin-film deposition and thus can complement the high-resolution, subtractive reverse stamping process described above.

To evaluate the impact of the physical surface modification, Figure 4 (a)-(b) compare EGaIn wetting properties on a smooth PDMS surface and a paper-textured PDMS surface; clearly, uniform EGaIn films can be patterned on the paper-textured PDMS substrate, while non-uniform EGaIn droplets form on the smooth PDMS substrate. The additive stamping process decreases the surface roughness $\left(R_{\mathrm{a}}\right)$ with increasing number of stamping steps $(n)$, which indicates that the stamped EGaIn film is getting smoother and uniformly molded $(t \approx 1.45 \mu \mathrm{m}$ at $n=5)$ on the physical-surface-modified PDMS substrate (Figure 4 (c)-(d)).

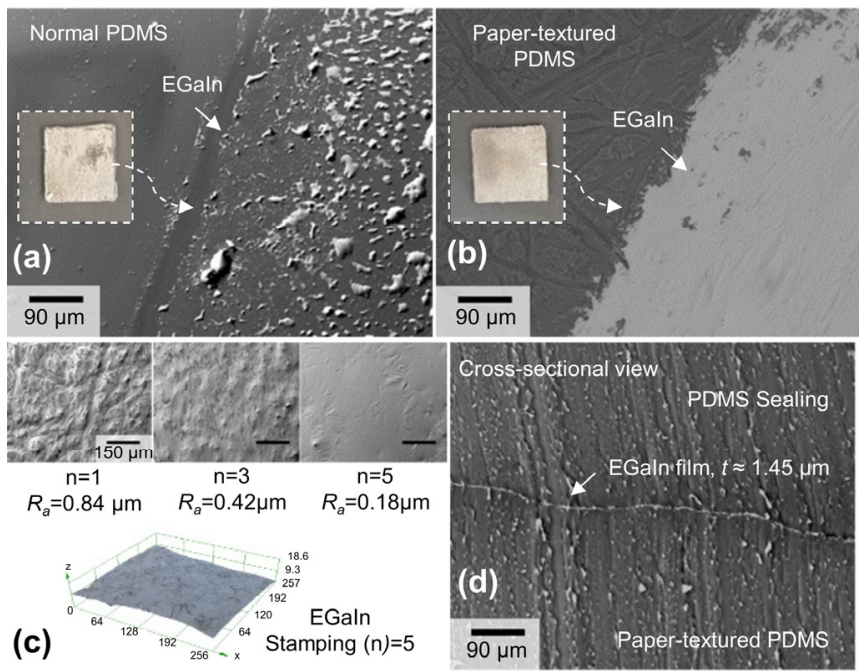

Figure 4: Patterned EGaIn films on (a) a normal, smooth PDMS surface and (b) a paper-textured PDMS surface; (c) surface characteristics of EGaIn thin films as a function of the number of stamping steps (n); (d) cross-sectional view of patterned EGaIn film on paper-textured PDMS after sealing with an additional PDMS layer. 
Electrical and Mechanical Characteristics of Fabricated Soft Electronic Devices Using Additive Stamping Process

Using the additive stamping process, soft passive components and circuits were designed and fabricated. Figure 5 (a) shows fabricated soft passive components, such as a resistor $\left(R_{\text {meas }}=16.3\right.$ $\Omega)$, a planar spiral inductor $\left(L_{\text {meas }}=43.7 \mathrm{nH}\right)$, and an interdigitated capacitor $\left(C_{\text {meas }}=1.48 \mathrm{pF}\right)$, all having $1 \mathrm{~mm}$ line width and $1 \mathrm{~mm}$ line spacing, attached to a non-planar object. One of the main requirements for soft microsystems is to maintain their electronic functionality during mechanical deformation. Thus, we experimentally investigated the electrical functionality by applying bending and stretching forces. Figure 5 (b) shows a simple circuit comprising a $5 \times 5$ array of light emitting diodes (LEDs). The commercial surface-mount LEDs were manually attached to EGaIn contacts along the patterned EGaIn lines using a tweezer and a stereo microscope for alignment and subsequently sealed using an additional PDMS casting step. Applying a constant current to the soft circuit, the LED array was subjected to bending (bending radius, $r=10 \mathrm{~mm}$ ) and stretching (strain, $\varepsilon \leq 50 \%$ ) deformation. The soft circuit withstood these bending deformations and strain up to $50 \%$ while maintaining its electrical functionality, which also confirmed that the EGaIn thin film was uniformly patterned without voids.

(a)

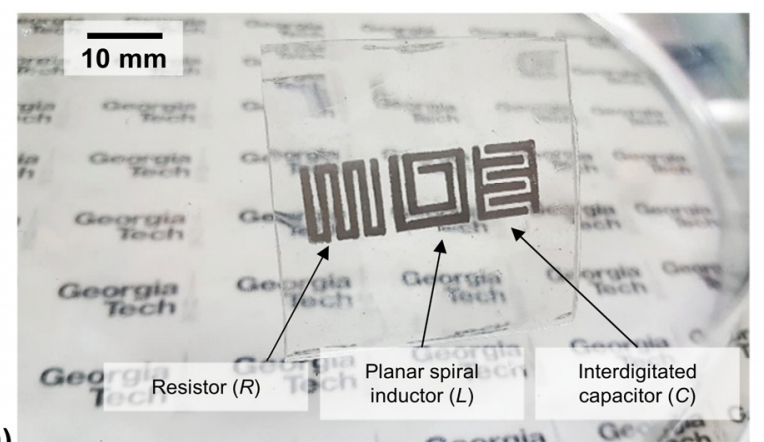

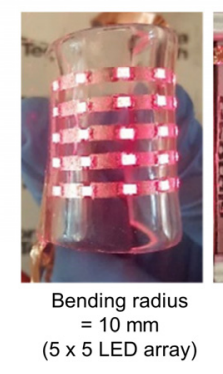

(b)

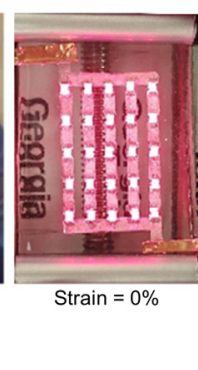

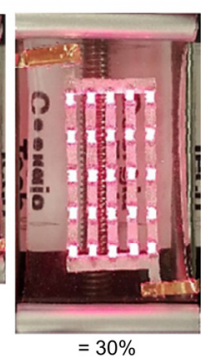

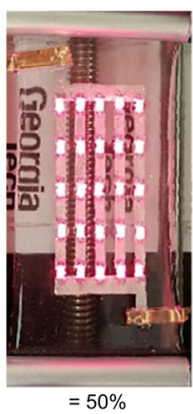

Figure 5: (a) Fabricated soft passive components using the additive stamping process and (b) soft circuit with embedded LEDs $(5 \times 5$ array) operated under bending (bending radius $=10 \mathrm{~mm}$ ) and stretching (strain $\leq 50 \%$ ) deformation.

\section{SOFT BIOLOGICAL MICROSYSTEMS}

To highlight the multiscale EGaIn patterning capabilities, a fingertip-mountable, soft and 3D-integrated biological sensing platform, comprising a soft sensing layer with an integrated pulse oximeter and a soft interfacing circuit layer, is demonstrated for non-invasive and real-time heart rate and blood oxygen monitoring (Figure 6 (a)). The soft sensor layer is fabricated using the subtractive reverse stamping technique for micro-scale EGaIn patterning to connect an integrated pulse oximeter (MAX30100, Maxim Integrated Products Inc.), while the additive stamping technique is utilized to fabricate the soft printed circuit board
(sPCB) for the interfacing circuit (Figure 6 (b)-(d)). The soft sensor and circuit layers are then vertically interconnected through EGaIn soft vias to form a 3D-integrated soft system. Figure 7 shows the measured photoplethysmogram (PPG) waveforms of the infrared (IR) LED and the red LED, as well as the extracted heart rate (HR) and saturation of peripheral oxygen $\left(\mathrm{SpO}_{2}\right)$ measured using the PDMS/EGaIn-based soft sensing system $(\mathrm{HR}=79 \pm 3.6 \mathrm{bpm}$ and $\mathrm{SpO}_{2}=97 \%$ ). The results are compared to results obtained using a PCB-based rigid sensing system (MIKROE-2000, Mikro Elektronika Ltd., $\mathrm{HR}=79.1 \pm 3.1 \mathrm{bpm}$ and $\mathrm{SpO}_{2}=97 \%$ ), demonstrating identical sensing performance of the soft microsystem.

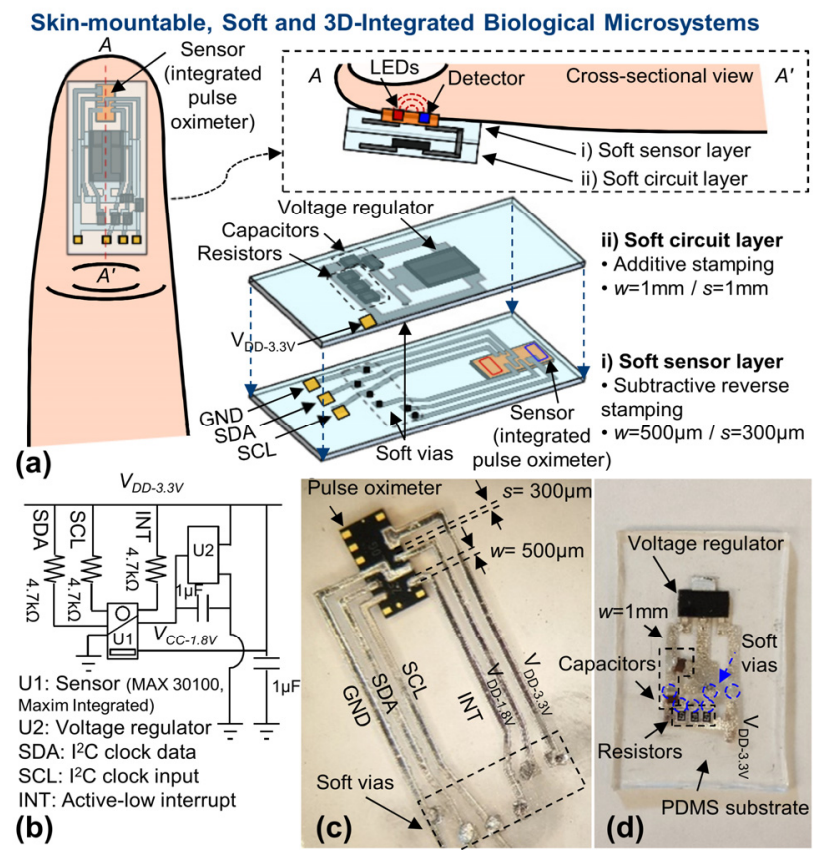

Figure 6: (a) Soft and 3D-integrated biological microsystem using a pulse oximeter mounted on a fingertip for heart rate and blood oxygen monitoring; (b) circuit diagram; fabrication and electric component integration of (c) soft sensor layer patterned using subtractive reverse stamping process and (d) soft circuit layer patterned using additive stamping process.
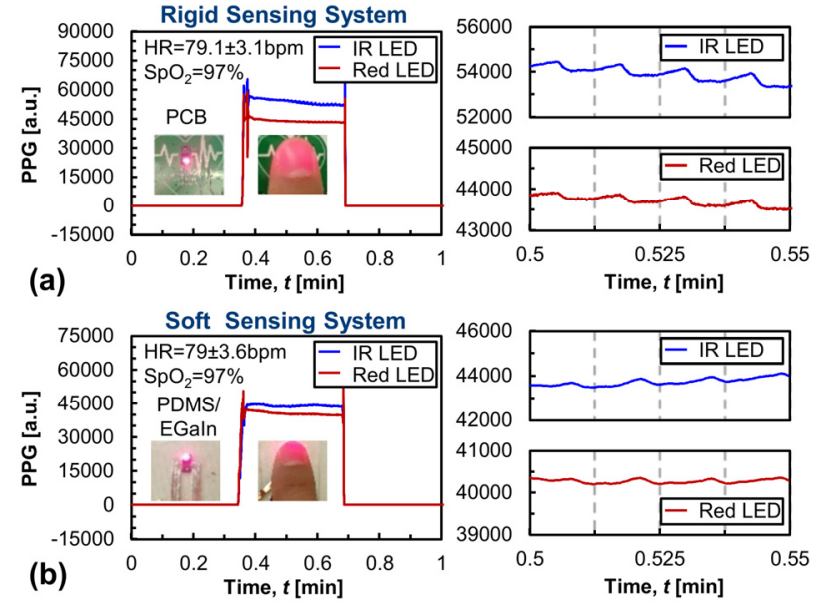

Figure 7: Measured photoplethysmogram (PPG) waveforms of infrared (IR) LED and red LED using (a) PCB-based rigid sensing system (heart rate $(H R)=79.1 \pm 3.1 \mathrm{bpm}$ and saturation of peripheral oxygen $\left(\mathrm{SpO}_{2}\right)=97 \%$ ) and (b) PDMS/EGaIn-based soft sensing system $\left(\mathrm{HR}=79 \pm 3.6 \mathrm{bpm}\right.$ and $\left.\mathrm{SpO}_{2}=97 \%\right)$. 


\section{CONCLUSION}

This paper reports multiscale EGaIn thin-film patterning based on soft lithography by utilizing a subtractive reverse stamping approach for micro-scale and high-density patterns and an additive stamping approach for centimeter-scale, large-area patterns. By combining these complementary patterning techniques, fabricated soft electronic components can be vertically integrated to form high-density and multifunctional soft microsystems for physical, chemical, and biological sensing applications.

\section{ACKNOWLEDGEMENT}

This work was performed in part at the Georgia Tech Institute for Electronics and Nanotechnology (IEN), a member of the National Nanotechnology Coordinated Infrastructure, which is supported by the National Science Foundation (Grant ECCS-1542174).

\section{REFERENCES}

[1] A. Thomas, "Functional materials: From hard to soft porous frameworks," Angewandte Chemie International Edition, 49, 8328 (2010).

[2] R. L. Truby and J. A. Lewis, "Printing soft matter in three dimensions," Nature, 540, 371 (2016).

[3] A. Chortos and Z. Bao, "Skin-inspired electronic devices," Materials Today, 17, 321 (2014).

[4] M. D. Dickey, "Stretchable and soft electronics using liquid metals," Advanced Materials, 29, 1606425 (2017).

[5] N. Kazem, T. Hellebrekers, and C. Majidi, "Soft multifunctional composites and emulsions with liquid metals," Advanced Materials, 29, 1605985 (2017).

[6] M. D. Dickey, R. C. Chiechi, R. J. Larsen, E. A. Weiss, D. A. Weitz, and G. M. Whitesides, "Eutectic gallium-indium (egain): A liquid metal alloy for the formation of stable structures in microchannels at room temperature," Advanced Functional Materials, 18, 1097 (2008).

[7] M. D. Dickey, "Emerging applications of liquid metals featuring surface oxides," ACS Applied Materials \& Interfaces, 6, 18369 (2014).

[8] S. H. Jeong, A. Hagman, K. Hjort, M. Jobs, J. Sundqvist, and $\mathrm{Z}$. Wu, "Liquid alloy printing of microfluidic stretchable electronics," Lab on a Chip, 12, 4657 (2012).

[9] N. Lazarus, S. S. Bedair, and I. M. Kierzewski, "Ultrafine pitch stencil printing of liquid metal alloys," ACS Applied Materials \& Interfaces, 9, 1178 (2017).

[10] P. Yong-Lae, C. Bor-Rong, and R. J. Wood, "Design and fabrication of soft artificial skin using embedded microchannels and liquid conductors," IEEE Sensors Journal, 12, 2711 (2012).

[11] Y. Lin, O. Gordon, M. R. Khan, N. Vasquez, J. Genzer, and M. D. Dickey, "Vacuum filling of complex microchannels with liquid metal," Lab on a Chip, 17, 3043 (2017).

[12] J. W. Boley, E. L. White, G. T. C. Chiu, and R. K. Kramer, "Direct writing of gallium-indium alloy for stretchable electronics," Advanced Functional Materials, 24, 3501 (2014).

[13] C. Ladd, J. H. So, J. Muth, and M. D. Dickey, "3d printing of free standing liquid metal microstructures," Advanced Materials, 25, 5081 (2013).

[14] M. R. Khan, C. B. Eaker, E. F. Bowden, and M. D. Dickey, "Giant and switchable surface activity of liquid metal via surface oxidation," Proceedings of the National Academy of Sciences of the United States of America, 111, 14047 (2014).
T. Lu, E. J. Markvicka, Y. Jin, and C. Majidi, "Soft-matter printed circuit board with uv laser micropatterning," ACS Applied Materials \& Interfaces, 9, 22055 (2017).

[16] B. A. Gozen, A. Tabatabai, O. B. Ozdoganlar, and C. Majidi, "High-density soft-matter electronics with micron-scale line width," Advanced Materials, 26, 5211 (2014).

[17] I. D. Joshipura, H. R. Ayers, C. Majidi, and M. D. Dickey, "Methods to pattern liquid metals," Journal of Materials Chemistry C, 3, 3834 (2015).

[18] M. A. H. Khondoker and D. Sameoto, "Fabrication methods and applications of microstructured gallium based liquid metal alloys," Smart Materials and Structures, 25, 093001 (2016).

[19] J. A. Rogers and R. G. Nuzzo, "Recent progress in soft lithography," Materials Today, 8, 50 (2005).

[20] D. Qin, Y. Xia, and G. M. Whitesides, "Soft lithography for micro- and nanoscale patterning," Nature Protocols, 5, 491 (2010).

[21] M.-g. Kim, H. Alrowais, S. Pavlidis, and O. Brand, "Size-scalable and high-density liquid-metal-based soft electronic passive components and circuits using soft lithography," Advanced Functional Materials, 27, 1604466 (2017).

[22] M.-g. Kim, H. Alrowais, S. Pavlidis, and Oliver Brand* "Scalable liquid metal thin line patterning for passive electronic components using soft lithography," in The 16th Solid-State Sensors, Actuators and Microsystems Workshop, Hilton Head, USA, 2016, pp. 62-63.

[23] M.-g. Kim, H. Alrowais, C. Kim, P. Yeon, M. Ghovanloo, and O. Brand, "All-soft, battery-free, and wireless chemical sensing platform based on liquid metal for liquid- and gas-phase voc detection," Lab on a Chip, 17 , 2323 (2017).

[24] M.-g. Kim, H. Alrowais, and O. Brand, "3d-integrated and multifunctional all-soft physical microsystems based on liquid metal for electronic skin applications," Advanced Electronic Materials, 4, 1700434 (2018).

[25] M.-g. Kim, H. Alrowais, and O. Brand, "All-soft physical and chemical microsystems based on liquid metal for wearable electronics applications," in The 30th international conference on Micro Electro Mechanical Systems, Las Vegas, USA, 2017, pp. 1162-1165.

[26] G. Li, M. Parmar, and D. W. Lee, "An oxidized liquid metal-based microfluidic platform for tunable electronic device applications," Lab on a Chip, 15, 766 (2015).

\section{CONTACT}

M. Kim, tel: +1-404-317-4892; mgk@gatech.edu

O. Brand, tel:+1-404-894-9425; oliver.brand@ece.gatech.edu 